PROCEEDINGS OF THE

AMERICAN MATHEMATICAL SOCIETY

Volume 137, Number 6, June 2009, Pages 1945-1950

S 0002-9939(08)09759-1

Article electronically published on December 17, 2008

\title{
ASYMPTOTIC DEPTH OF TWISTED HIGHER DIRECT IMAGE SHEAVES
}

\author{
RENATE BÄR AND MARKUS BRODMANN
}

(Communicated by Bernd Ulrich)

\begin{abstract}
Let $\pi: X \rightarrow X_{0}$ be a projective morphism of schemes, such that $X_{0}$ is Noetherian and essentially of finite type over a field $K$. Let $i \in \mathbb{N}_{0}$, let $\mathcal{F}$ be a coherent sheaf of $\mathcal{O}_{X}$-modules and let $\mathcal{L}$ be an ample invertible sheaf over $X$. Let $Z_{0} \subseteq X_{0}$ be a closed set. We show that the depth of the higher direct image sheaf $\mathcal{R}^{i} \pi_{*}\left(\mathcal{L}^{n} \otimes_{\mathcal{O}_{X}} \mathcal{F}\right)$ along $Z_{0}$ ultimately becomes constant as $n$ tends to $-\infty$, provided $X_{0}$ has dimension $\leq 2$. There are various examples which show that the mentioned asymptotic stability may fail if $\operatorname{dim}\left(X_{0}\right) \geq 3$. To prove our stability result, we show that for a finitely generated graded module $M$ over a homogeneous Noetherian ring $R=\bigoplus_{n>0} R_{n}$ for which $R_{0}$ is essentially of finite type over a field and an ideal $\mathfrak{a}_{0} \subseteq R_{0}$, the $\mathfrak{a}_{0}$-depth of the $n$-th graded component $H_{R_{+}}^{i}(M)_{n}$ of the $i$-th local cohomology module of $M$ with respect to $R_{+}:=\bigoplus_{k>0} R_{k}$ ultimately becomes constant in codimension $\leq 2$ as $n$ tends to $-\infty$.
\end{abstract}

\section{INTRODUCTION}

Let $\pi: X \rightarrow X_{0}$ be a projective morphism of schemes, such that $X_{0}$ is Noetherian and essentially of finite type over a field. Let $\mathcal{F}$ be a coherent sheaf of $\mathcal{O}_{X}$-modules and let $\mathcal{L}$ be an ample invertible sheaf of $\mathcal{O}_{X}$-modules. Let $i \in \mathbb{N}_{0}$. In [2, Theorem 5.5] we did show:

(1.1) As $n \rightarrow-\infty$, the set

$$
\begin{aligned}
& \operatorname{Ass}_{X_{0}}\left(\mathcal{R}^{i} \pi_{*}\left(\mathcal{L}^{n} \otimes_{\mathcal{O}_{X}} \mathcal{F}\right)\right) \leq 2 \\
& :=\left\{x_{0} \in \operatorname{Ass}_{X_{0}}\left(\mathcal{R}^{i} \pi_{*}\left(\mathcal{L}^{n} \otimes_{\mathcal{O}_{X}} \mathcal{F}\right)\right) \mid \operatorname{dim}\left(\mathcal{O}_{X_{0}, x_{0}}\right) \leq 2\right\}
\end{aligned}
$$

ultimately becomes constant.

Our aim is to prove a corresponding but stronger stability result for the depths in codimension $\leq 2$ of the sheaves $\mathcal{R}^{i} \pi_{*}\left(\mathcal{L}^{n} \otimes_{\mathcal{O}_{X}} \mathcal{F}\right)$ along a closed subset $Z_{0} \subseteq X_{0}$.

To make this precise we introduce the following notion: If $Z_{0} \subseteq X_{0}$ is a closed set, $\mathcal{G}$ is a coherent sheaf of $\mathcal{O}_{X_{0}}$-modules and $t \in \mathbb{N}_{0}$, we define the depth of $\mathcal{G}$ along $Z_{0}$ and the depth in codimension $\leq t$ of $\mathcal{G}$ along $Z_{0}$ respectively by

(1.2) $\operatorname{depth}\left(Z_{0}, \mathcal{G}\right):=\inf \left\{\operatorname{depth}\left(\mathcal{G}_{x_{0}}\right) \mid x_{0} \in Z_{0}\right\}$

(1.3) $\operatorname{depth}\left(Z_{0}, \mathcal{G}\right) \leq t:=\inf \left\{\operatorname{depth}\left(\mathcal{G}_{x_{0}}\right) \mid x_{0} \in Z_{0}, \operatorname{dim}\left(\mathcal{O}_{X_{0}, x_{0}}\right) \leq t\right\}$

Received by the editors April 23, 2008, and, in revised form, August 26, 2008.

2000 Mathematics Subject Classification. Primary 13D45, 13E10, 14 F05.

Key words and phrases. Local cohomology, graded modules, depth, projective schemes, ample invertible sheaves, higher direct images.

(C)2008 American Mathematical Society Reverts to public domain 28 years from publication 
We use the convention that $\inf (\emptyset):=\infty$, so that

$$
\operatorname{depth}\left(Z_{0}, \mathcal{G}\right)^{\leq t}=\infty \text { if } \operatorname{codim}\left(Z_{0}, X_{0}\right)>t .
$$

Our main result now says (cf. Theorem 3.5)

(1.4) As $n \rightarrow-\infty$, the number

$$
\operatorname{depth}\left(Z_{0}, \mathcal{R}^{i} \pi_{*}\left(\mathcal{L}^{n} \otimes_{\mathcal{O}_{X}} \mathcal{F}\right)\right)^{\leq 2}
$$

ultimately becomes constant.

As an immediate consequence we obtain (cf. Corollary 3.6)

(1.5) Let $\operatorname{dim}\left(X_{0}\right) \leq 2$. Then, as $n \rightarrow-\infty$, the number

$$
\operatorname{depth}\left(Z_{0}, \mathcal{R}^{i} \pi_{*}\left(\mathcal{L}^{n} \otimes_{\mathcal{O}_{X}} \mathcal{F}\right)\right)
$$

ultimately becomes constant.

The examples constructed by Chardin-Cutkosky-Herzog-Srinivasan [5] illustrate that the conclusion of (1.5) need not hold if $\operatorname{dim}\left(X_{0}\right) \geq 3$.

The basic tool to prove our main result is a corresponding stability result for the depths of graded components of certain local cohomology modules. We shall establish this result in the next section.

\section{Depth and local Cohomology}

By $\mathbb{N}_{0}$ we denote the set of non-negative integers, and by $\mathbb{N}$ we denote the set of positive integers.

Notation and Conventions 2.1. (A) Throughout this paper let $R=R_{0} \oplus R_{1} \oplus$ $\cdots$ be a standard graded ring. So, $R$ is $\mathbb{N}_{0}$-graded, $R_{0}$ is Noetherian and there are finitely many elements $a_{1}, \ldots, a_{k} \in R_{1}$ such that $R=R_{0}\left[a_{1}, \ldots, a_{k}\right]$. By $R_{+}$we denote the irrelevant ideal of $R$; thus $R_{+}=R_{1} \oplus R_{2} \oplus \cdots$.

(B) If $i \in \mathbb{N}_{0}$ and $M$ is a graded $R$-module, we write $H_{R_{+}}^{i}(M)$ for the $i$-th local cohomology module of $M$ with respect to $R_{+}$, and we always furnish this module with its natural grading.

For $n \in \mathbb{Z}$ we denote by $H_{R_{+}}^{i}(M)_{n}$ the $n$-th graded component of $H_{R_{+}}^{i}(M)$. Keep in mind that $H_{R_{+}}^{i}(M)_{n}$ is a finitely generated $R_{0}$-module for all $n \in \mathbb{Z}$ and vanishes for all $n \gg 0$, provided that the graded $R$-module $M$ is finitely generated.

(C) Now, fix an ideal $\mathfrak{a}_{0} \subseteq R_{0}$. We write $\operatorname{Var}\left(\mathfrak{a}_{0}\right)$ for the variety $\left\{\mathfrak{p}_{0} \in \operatorname{Spec}\left(R_{0}\right) \mid\right.$ $\left.\mathfrak{a}_{0} \subseteq \mathfrak{p}_{0}\right\}$ of $\mathfrak{a}_{0}$. Keep in mind that for a finitely generated $R_{0}$-module $T$ we always have

$$
\operatorname{depth}\left(\mathfrak{a}_{0}, T\right)=\inf \left\{\operatorname{depth}\left(T_{\mathfrak{p}_{0}}\right) \mid \mathfrak{p}_{0} \in \operatorname{Var}\left(\mathfrak{a}_{0}\right)\right\} .
$$

Now, for any finitely generated $R_{0}$-module $T$ and any $t \in \mathbb{N}_{0}$ we define the depth in codimension $\leq t$ of $T$ with respect to $\mathfrak{a}_{0}$ by

$$
\operatorname{depth}\left(\mathfrak{a}_{0}, T\right)^{\leq t}=\inf \left\{\operatorname{depth}\left(T_{\mathfrak{p}_{0}}\right) \mid \mathfrak{p}_{0} \in \operatorname{Var}\left(\mathfrak{a}_{0}\right), \operatorname{height}\left(\mathfrak{p}_{0}\right) \leq t\right\} .
$$

Again we use the convention that $\inf (\emptyset)=\infty$, so that

$$
\operatorname{depth}\left(\mathfrak{a}_{0}, T\right)^{\leq t} \in\{0,1, \ldots, t, \infty\}
$$

with

$$
\operatorname{depth}\left(\mathfrak{a}_{0}, T\right)^{\leq t}=\infty \Longleftrightarrow \forall \mathfrak{p}_{0} \in \operatorname{Var}\left(\mathfrak{a}_{0}\right) \cap \operatorname{Supp}(T): \operatorname{height}\left(\mathfrak{p}_{0}\right)>t .
$$


(D) We say that a graded $R$-module $U=\bigoplus_{n \in \mathbb{Z}} U_{n}$ is tame if

$$
U_{n}=0 \text { for all } n \ll 0 \text { or } U_{n} \neq 0 \text { for all } n \ll 0 \text {. }
$$

(E) Let $\left(\mathcal{S}_{n}\right)_{n \in \mathbb{Z}}$ be a family of numbers or sets. We say that $\mathcal{S}_{n}$ is asymptotically stable for $n \rightarrow-\infty$ if there is some $n_{0} \in \mathbb{Z}$ such that $\mathcal{S}_{n}=\mathcal{S}_{n_{0}}$ for all $n \leq n_{0}$.

Lemma 2.2. Assume that $\left(R_{0}, \mathfrak{m}_{0}\right)$ is local and of dimension $\leq 2$. Let $i \in \mathbb{N}_{0}$ and let $M$ be a finitely generated graded $R$-module such that $\operatorname{Ass}_{R_{0}}\left(H_{R_{+}}^{i}(M)_{n}\right)$ is asymptotically stable for $n \rightarrow-\infty$.

Then $\operatorname{depth}_{R_{0}}\left(H_{R_{+}}^{i}(M)_{n}\right)$ is asymptotically stable for $n \rightarrow-\infty$.

Proof. By the asymptotic stability of $\operatorname{Ass}_{R_{0}}\left(H_{R_{+}}^{i}(M)_{n}\right)$ for $n \rightarrow-\infty$ we have

$$
\begin{gathered}
\operatorname{depth}_{R_{0}}\left(H_{R_{+}}^{i}(M)_{n}\right)=0 \text { for all } n \ll 0, \text { or else } \\
\operatorname{depth}_{R_{0}}\left(H_{R_{+}}^{i}(M)_{n}\right)>0 \text { for all } n \ll 0 .
\end{gathered}
$$

In the first case we are done. So, assume that we are in the second case.

According to [3, Proposition 5.10] the graded $R$-module $H_{\mathfrak{m}_{0} R}^{1}\left(H_{R_{+}}^{i}(M)\right)$ is Artinian and hence tame. Therefore either

$$
\begin{gathered}
H_{\mathfrak{m}_{0}}^{1}\left(H_{R_{+}}^{i}(M)_{n}\right)=0 \text { for all } n \ll 0 \text { or else } \\
H_{\mathfrak{m}_{0}}^{1}\left(H_{R_{+}}^{i}(M)_{n}\right) \neq 0 \text { for all } n \ll 0 .
\end{gathered}
$$

As $\operatorname{dim}\left(R_{0}\right) \leq 2$ we thus respectively have either

$$
\begin{gathered}
\operatorname{depth}_{R_{0}}\left(H_{R_{+}}^{i}(M)_{n}\right)=2 \text { for all } n \ll 0 \text { or } \\
\operatorname{depth}_{R_{0}}\left(H_{R_{+}}^{i}(M)_{n}\right)=1 \text { for all } n \ll 0 .
\end{gathered}
$$

Proposition 2.3. Let $\mathfrak{a}_{0} \subseteq R_{0}$ be an ideal, let $i \in \mathbb{N}_{0}$ and let $M$ be a finitely generated graded $R$-module such that $\operatorname{Ass}_{R_{0}}\left(H_{R_{+}}^{i}(M)_{n}\right)^{\leq 2}$ is asymptotically stable for $n \rightarrow-\infty$.

Then $\operatorname{depth}\left(\mathfrak{a}_{0}, H_{R_{+}}^{i}(M)_{n}\right) \leq 2$ is asymptotically stable for $n \rightarrow-\infty$.

Proof. Let $t:=\liminf _{n \rightarrow-\infty} \operatorname{depth}\left(\mathfrak{a}_{0}, H_{R_{+}}^{i}(M)_{n}\right) \leq 2$. Observe that $t \in\{0,1,2, \infty\}$ (cf. 2.1 (C) $)$. We have to show that $t(n):=\operatorname{depth}\left(\mathfrak{a}_{0}, H_{R_{+}}^{i}(M)_{n}\right)^{\leq 2}=t$ for all $n \ll 0$.

If $t=\infty$, this is clear. So, let $t \in\{0,1,2\}$. We set

$$
V:=\left\{\mathfrak{p}_{0} \in \operatorname{Var}\left(\mathfrak{a}_{0}\right) \mid \operatorname{height}\left(\mathfrak{p}_{0}\right) \leq 2\right\} .
$$

By our hypothesis the set $V \cap \operatorname{Ass}_{R_{0}}\left(H_{R_{+}}^{i}(M)_{n}\right)$ takes a constant value $U$ for all $n \ll 0$. If $U \neq \emptyset$, we have $\operatorname{depth}\left(\mathfrak{a}_{0}, H_{R_{+}}^{i}(M)_{n}\right)^{\leq 2}=0$ for all $n \ll 0$. If $U=\emptyset$, we have $\operatorname{depth}\left(\mathfrak{a}_{0}, H_{R_{+}}^{i}(M)_{n}\right)^{\leq 2}>0$ for all $n \ll 0$. This gives our claim if $t=0$.

So, assume that $t \in\{1,2\}$. Then $\operatorname{depth}\left(\mathfrak{a}_{0}, H_{R_{+}}^{i}(M)_{n}\right) \leq 2 \geq 1$ for all $n \ll 0$. By our hypothesis, the set $V \cap \operatorname{Supp}_{R_{0}}\left(H_{R_{+}}^{i}(M)_{n}\right)=V \cap \overline{\operatorname{Ass}_{R_{0}}\left(H_{R_{+}}^{i}(M)_{n}\right)}$ takes a constant value $W$ for all $n \ll 0$. If height $\left(\mathfrak{p}_{0}\right) \leq 1$ for some $\mathfrak{p}_{0} \in W$, we have $\operatorname{depth}\left(\mathfrak{a}_{0}, H_{R_{+}}^{i}(M)_{n}\right) \leq \operatorname{height}\left(\mathfrak{p}_{0}\right) \leq 1$ for all $n \ll 0$, so that $\operatorname{depth}\left(\mathfrak{a}_{0}, H_{R_{+}}^{i}(M)_{n}\right)$ $=1$ for all $n \ll 0$. Thus, our claim follows in this case.

Therefore, we may assume that height $\left(\mathfrak{p}_{0}\right)=2$ for all $\mathfrak{p}_{0} \in W$. As $W$ is closed in $V$ and $V$ is closed under generalization, the set $W$ must be finite. As $t<\infty$ we must have $\operatorname{depth}\left(\mathfrak{a}_{0}, H_{R_{+}}^{i}(M)_{n}\right) \leq 2<\infty$ for infinitely many $n<0$. Therefore $W \neq \emptyset$ (cf. $2.1(\bar{C})$ ) . 
Now by Lemma 2.2 and the Flat Base-Change Property of local cohomology (cf. [4, Theorem 13.1.8]) we get that $\operatorname{depth}_{\left(R_{0}\right)_{\mathfrak{p}_{0}}}\left(\left(H_{R_{+}}^{i}(M)_{n}\right)_{\mathfrak{p}_{0}}\right)$ is asymptotically stable for $n \rightarrow-\infty$ for all $\mathfrak{p}_{0} \in W$. As $W$ is finite it follows that $\operatorname{depth}\left(\mathfrak{a}_{0}, H_{R_{+}}^{i}(M)_{n}\right)=$ $\min \left\{\operatorname{depth}_{\left(R_{0}\right)_{\mathfrak{p}_{0}}}\left(\left(H_{R_{+}}^{i}(M)_{n}\right)_{\mathfrak{p}_{0}}\right) \mid \mathfrak{p}_{0} \in W\right\}$ is asymptotically stable for $n \rightarrow-\infty$.

Corollary 2.4. Assume that $R_{0}$ is essentially of finite type over a field. Let $i \in \mathbb{N}_{0}$, let $\mathfrak{a}_{0} \subseteq R_{0}$ be an ideal and let $M$ be a finitely generated graded $R$-module.

Then $\operatorname{depth}\left(\mathfrak{a}_{0}, H_{R_{+}}^{i}(M)_{n}\right) \leq 2$ is asymptotically stable for $n \rightarrow-\infty$.

Proof. According to [2, Proposition 3.5] the set $\operatorname{Ass}_{R_{0}}\left(H_{R_{+}}^{i}(M)_{n}\right)^{\leq 2}$ is asymptotically stable for $n \rightarrow-\infty$. Now, we may conclude by Proposition 2.3

Corollary 2.5. Assume that $\operatorname{dim}\left(R_{0}\right) \leq 2$ and $R_{0}$ is essentially of finite type over a field. Let $i \in \mathbb{N}_{0}$, let $\mathfrak{a}_{0} \subseteq R_{0}$ be an ideal and let $M$ be a finitely generated graded $R$-module.

Then $\operatorname{depth}\left(\mathfrak{a}_{0}, H_{R_{+}}^{i}(M)_{n}\right)$ is asymptotically stable for $n \rightarrow-\infty$.

This result actually is shown in 1 .

Remark 2.6. (A) According to [5], there is a normal homogeneous domain $R=$ $R_{0} \oplus R_{1} \oplus \cdots$, such that $\left(R_{0}, \mathfrak{m}_{0}\right)$ is local of dimension 3 , essentially of finite type over $\mathbb{C}$ and such that $H_{R_{+}}^{2}(R)$ is not tame. In particular $\operatorname{depth}\left(\mathfrak{m}_{0}, H_{R_{+}}^{2}(M)_{n}\right)$ is not asymptotically stable for $n \rightarrow-\infty$. So in codimensions $\geq 3$ the depth of $H_{R_{+}}^{i}(M)_{n}$ need not be asymptotically stable.

(B) Let $R=R_{0} \oplus R_{1} \oplus \cdots$ be as in $2.1(\underline{\mathrm{A}})$, let $\mathfrak{a}_{0} \subseteq R_{0}$ be an ideal and let $M$ be a finitely generated graded $R$-module. Let

$$
c:=\sup \left\{i \in \mathbb{N}_{0} \mid H_{R_{+}}^{i}(M) \neq 0\right\}
$$

be the cohomological dimension of $M$ with respect to $R_{+}$and let

$$
f:=\inf \left\{i \in \mathbb{N} \mid H_{R_{+}}^{i}(M) \text { not finitely generated }\right\}
$$

be the cohomological finiteness dimension of $M$ with respect to $R_{+}$.

If $f<\infty$, then clearly $f \leq c$. Moreover, it is well known that $\operatorname{Ass}_{R_{0}}\left(H_{R_{+}}^{c}(M)_{n}\right)$ need not be asymptotically stable for $n \rightarrow-\infty$ (cf. [8] for example) and that $\operatorname{Ass}_{R_{0}}\left(H_{R_{+}}^{f}(M)_{n}\right)$ is asymptotically stable for $n \rightarrow-\infty$. In [7] it is shown:

If $f=c$, then $\operatorname{depth}\left(\mathfrak{a}_{0}, H_{R_{+}}^{c}(M)_{n}\right)$ is asymptotically stable for $n \rightarrow-\infty$.

\section{DePth AND higher DiRECT IMAGeS}

For the unexplained terminology of this section we refer to 6 .

Notation and Conventions 3.1. (A) For the rest of this paper let $X_{0}$ denote a Noetherian scheme, let $\pi: X \rightarrow X_{0}$ denote a projective scheme over $X_{0}$ with very ample sheaf $\mathcal{O}_{X}(1)$ and let $Z_{0} \subseteq X_{0}$ be a closed set.

(B) If $\mathcal{G}$ is a coherent sheaf of $\mathcal{O}_{X_{0}}$-modules and $t \in \mathbb{N}_{0}$ we always use the notation introduced in (1.2) and (1.3). 
(C) If $X_{0}$ is affine and $I\left(Z_{0}\right) \subseteq \mathcal{O}\left(X_{0}\right)$ is the vanishing ideal of $Z_{0}$, we write

$$
\begin{gathered}
\operatorname{depth}\left(Z_{0}, T\right):=\operatorname{depth}\left(I\left(Z_{0}\right), T\right)=\operatorname{depth}\left(Z_{0}, \widetilde{T}\right) \text { and } \\
\operatorname{depth}\left(Z_{0}, T\right)^{\leq t}:=\operatorname{depth}\left(I\left(Z_{0}\right), T\right)^{\leq t}=\operatorname{depth}\left(Z_{0}, \widetilde{T}\right)^{\leq t}
\end{gathered}
$$

for each finitely generated $\mathcal{O}\left(X_{0}\right)$-module $T$.

Proposition 3.2. Assume that $X_{0}$ is affine and essentially of finite type over a field. Let $\mathcal{F}$ be a coherent sheaf of $\mathcal{O}_{X}$-modules and let $i \in \mathbb{N}_{0}$.

Then $\operatorname{depth}\left(Z_{0}, H^{i}(X, \mathcal{F}(n))\right)^{\leq 2}$ is asymptotically stable for $n \rightarrow-\infty$.

Proof. Let $R_{0}:=\mathcal{O}\left(X_{0}\right), \mathfrak{a}_{0}:=I\left(Z_{0}\right) \subseteq R_{0}$. Then, there is a homogeneous Noetherian $R_{0}$-algebra $R=R_{0} \oplus R_{1} \oplus \cdots$ with $X=\operatorname{Proj}(R)$ and $\mathcal{O}_{X}(1)=R(1)^{\sim}$. Moreover there is a finitely generated graded $R$-module $M$ such that $\mathcal{F}=\widetilde{M}$. Now, for each $n \in \mathbb{Z}$ the Serre-Grothendieck Correspondence gives rise to a short exact sequence of $R_{0}$-modules

$$
0 \rightarrow H_{R_{+}}^{0}(M)_{n} \rightarrow M_{n} \rightarrow H^{0}(X, \mathcal{F}(n)) \rightarrow H_{R_{+}}^{1}(M)_{n} \rightarrow 0
$$

and to isomorphisms of $R_{0}$-modules

$$
H^{j}(X, \mathcal{F}(n)) \cong H_{R_{+}}^{j+1}(M)_{n} \text { for all } j>0 .
$$

Therefore, our claim follows by Corollary 2.4.

Proposition 3.3. Assume that $X_{0}$ is affine and essentially of finite type over a field. Let $\mathcal{F}$ be a coherent sheaf of $\mathcal{O}_{X}$-modules, let $\mathcal{L}$ be an ample invertible sheaf of $\mathcal{O}_{X}$-modules and let $i \in \mathbb{N}_{0}$.

Then, $\operatorname{depth}\left(Z_{0}, H^{i}\left(X, \mathcal{L}^{n} \otimes_{\mathcal{O}_{X}} \mathcal{F}\right)\right) \leq 2$ is asymptotically stable for $n \rightarrow-\infty$.

Proof. This follows from Proposition 3.2 by essentially the same arguments as used in the proof of [2, Theorem 5.3].

Corollary 3.4. Let $X_{0}, \mathcal{L}, \mathcal{F}$ and $i$ be as in Proposition 3.3. Assume in addition that $\operatorname{dim}\left(X_{0}\right) \leq 2$.

Then, $\operatorname{depth}\left(Z_{0}, H^{i}\left(X, \mathcal{L}^{n} \otimes_{\mathcal{O}_{X}} \mathcal{F}\right)\right)$ is asymptotically stable for $n \rightarrow-\infty$.

Theorem 3.5. Let $X_{0}$ be essentially of finite type over a field. Let $\mathcal{L}$ be an ample invertible sheaf of $\mathcal{O}_{X}$-modules and let $\mathcal{F}$ be a coherent sheaf of $\mathcal{O}_{X}$-modules. Let $i \in \mathbb{N}_{0}$.

Then $\operatorname{depth}\left(Z_{0}, \mathcal{R}^{i} \pi_{*}\left(\mathcal{L}^{n} \otimes_{\mathcal{O}_{X}} \mathcal{F}\right)\right) \leq 2$ is asymptotically stable for $n \rightarrow-\infty$.

Proof. We may assume that $X_{0}$ is affine. Now, we can conclude by Proposition 3.3 as $\mathcal{R}^{i} \pi_{*}\left(\mathcal{L}^{n} \otimes_{\mathcal{O}_{X}} \mathcal{F}\right) \cong H^{i}\left(X, \mathcal{L}^{n} \otimes_{\mathcal{O}_{X}} \mathcal{F}\right)^{\sim}$.

Corollary 3.6. Let $X_{0}, \mathcal{L}, \mathcal{F}$ and $i$ be as in Theorem 3.5. Assume in addition that $\operatorname{dim}\left(X_{0}\right) \leq 2$.

Then $\operatorname{depth}\left(Z_{0}, \mathcal{R}^{i} \pi_{*}\left(\mathcal{L}^{n} \otimes_{\mathcal{O}_{X}} \mathcal{F}\right)\right)$ is asymptotically stable for $n \rightarrow-\infty$.

Remark 3.7. The observations made in Remark 2.6 (A) show that the conclusions of Corollaries 3.4 and 3.6 need not hold if $\operatorname{dim}\left(X_{0}\right) \geq 3$. 


\section{REFERENCES}

[1] Bär, R.: Asymptotische Stabilität von Tiefen lokaler Kohomologiemoduln und von Tiefen und assoziierten Punkten höherer direkter Bilder kohärenter Garben, Master Thesis, University of Zürich, 2007.

[2] Brodmann, M.: A cohomological stability result for projective schemes over surfaces, J. Reine Angew. Math. 606 (2007) 179-192. MR2337647

[3] Brodmann, M.; Rohrer, F.; Sazeedeh, R.: Multiplicities of graded components of local cohomology modules, Journal of Pure and Applied Algebra 197 (2005) 249-278. MR2123988 (2006c:13023)

[4] Brodmann, M.; Sharp, R. Y.: Local cohomology: An algebraic introduction with geometric applications, Cambridge Stud. Adv. Math. 60, Cambridge University Press, 1998. MR1613627 (99h:13020)

[5] Chardin, M.; Cutkosky, S. D.; Herzog, J.; Srinivasan, H.: Duality and tameness, Michigan Math. J. 57 (in honour of Mel Hochster) (2008) 137-156.

[6] Hartshorne, R.: Algebraic Geometry, Grad. Texts Math. 52, Springer-Verlag, New York, 1977. MR0463157 (57:3116)

[7] Hassanzadeh, S. H.; Jahangiri M.; Zakeri, H.: Asymptotic behaviour and Artinian property of graded local cohomology modules, preprint, 2008.

[8] Singh, A. K.; Swanson, I.: Associated primes of local cohomology modules and of Frobenius powers, Intern. Math. Res. Notices 33 (2004) 1703-1733. MR2058025 (2005d:13030)

Institut FÜr Mathematik, Universität ZÜrich, WinterthURERStrasse 190, CH-8057 ZÜrich, SWITZERLAND

Current address: Kapellenweg 5, CH-8572 Berg, Switzerland

E-mail address: renatebaer@gmx.ch

Institut Für Mathematik, Universität ZÜrich, WinterthURERStrasse 190, CH-8057 ZÜRICH, SWITZERLAND

E-mail address: brodmann@math.unizh.ch 